\title{
Features of infant exposure to tobacco smoke in a cohort study in Tasmania
}

\author{
Anne-Louise Ponsonby, David Couper, Terence Dwyer
}

\begin{abstract}
Study objectives - To document changes in smoking style around infants over time and to identify factors associated with the smoking hygiene of mothers and others. Design - A population based cohort study. Setting - Population based, involving $22 \%$ of live births in Tasmania, Australia.

Participants - From 1 May 1988 to 30 April, 1993, 6109 infants and their mothers (89\% of eligible infants) participated in the hospital and home interview of the cohort study. Infants eligible for cohort entry were those assessed at birth to be at a higher risk of SIDS.

Main results - The overall proportion of mothers who smoked during pregnancy and postnatally did not decline. Increasing trends were found for mothers and others not smoking in the same room as baby or while holding or feeding the baby, significant over the five year period. Good smoking hygiene (mother not smoking in the same room as baby) was positively associated with - first birth (OR $=1.74$ $(1 \cdot 30,2 \cdot 33))$, low birth weight $(1 \cdot 69(1 \cdot 27$, 2.23)), being born after 1 May $1991(1.67$ $(1 \cdot 33,2 \cdot 11))$, and private health insurance status $(1.39(1.02,1.90))$. Good smoking hygiene was negatively associated with maternal smoking during pregnancy $(0 \cdot 50$ $(0 \cdot 31,0 \cdot 80))$, intention to bottle feed $(0.62$ $(0.49,0.78))$, the level of maternal postnatal smoking, increasing numbers of smokers in the household, and parents cohabiting but unmarried. A similar analysis was conducted for other household residents who smoked.

Conclusions - Changes in maternal smoking prevalence have been small. The exposure of infants to tobacco smoke postnatally has decreased significantly, although a large proportion of infants are still exposed to tobacco smoke. The identification of the above parental and infant factors associated with good smoking hygiene should be useful for health education planning.
\end{abstract}

Menzies Centre for Population Health Research, 17 Liverpool Street, Hobart,

Tasmania 7000

A-L Ponsonby

D Couper

T Dwyer

Correspondence to: Dr A-L Ponsonby.

Accepted for publication August 1995

( $\mathcal{A}$ Epidemiol Community Health 1996;50:40-46)

Exposure to environmental tobacco smoke (ETS) is a serious public health problem. ${ }^{1}$ The US Environmental Protection Agency has reviewed the scientific evidence on the respiratory health effects of passive smoking. It concluded that, for children, ETS exposure is causally associated with an increased risk of lower respiratory tract infections, increased prevalence of fluid in the middle ear, symptoms of upper respiratory tract irritation, a reduction in lung function, and the precipitation or exacerbation of asthma in childhood. ${ }^{1}$ The role of passive smoking in the causation of asthma is unclear but at least three studies have suggested that children exposed to ETS may have a higher than average risk of asthma. ${ }^{2}$ Postnatal passive smoking by the infant also seems to increase the risk of SIDS in addition to the adverse effect of smoking during pregnancy on SIDS. ${ }^{34}$ The American Academy of Pediatrics has recently recommended that a tobacco free environment should be an imperative for the health of children and adolescents. ${ }^{5}$

To achieve the goal of a tobacco free environment, it is important to identify the characteristics of smokers with poor smoking hygiene and to monitor smoking hygiene trends over time. Children whose parents smoke have often also been exposed to smoke during fetal development, making it difficult to separate these two periods of passive smoking. ${ }^{6}$ Most studies on the effect of passive smoking in the home have related mother's smoking to the child's health problems. However, the physical distance between the new baby and the smoking mother or other family members will also correlate with the amount of cotinine in the baby's urine in addition to the amount smoked. ${ }^{78}$ This means that studies investigating the relationship between parental smoking and child health should also examine parental smoking hygiene.

Here we examine the smoking and smoking hygiene patterns of mothers and others in an infant cohort study. We examine changes in smoking style over time, the association of good smoking hygiene with maternal, infant and environmental factors, and the relationship between smoking during pregnancy and postnatal smoking hygiene after controlling for relevant confounding factors, including the amount smoked.

\section{Methods}

An ongoing prospective cohort study was begun in 1988 to investigate the aetiology of SIDS. It involves the six major obstetric hospitals in Tasmania, representing approximately $93 \%$ of live births in this state. Infants born within these hospitals are assessed using a scoring system to predict those at higher risk of SIDS. The development and performance of this model is described elsewhere. ${ }^{9}$ The infants are 
given a composite score based on materna age, birth weight, season of birth, infant sex, duration of the second stage of labour, and intended infant feeding. Infants with a score over a certain cut off point are eligible to join the study. All multiple births are also included in the study. Infants with severe neonatal disease or a major congenital anomaly, infants who would not be resident in Tasmania at one month of age, and infants for adoption are excluded from the study.

Standard study measurements were collected by research assistants in three stages. Firstly, a hospital interview was conducted when the infant was four days of age. Sociodemographic, obstetric, and perinatal data, including information on maternal smoking practice, nutrition, and alcohol during pregnancy were obtained. The data on antenatal smoking included maternal active cigarette smoking by trimester, whether the mother lived with a smoker while pregnant, and average exposure to tobacco smoke inside and outside the home in terms of approximate number of cigarettes per day and minutes exposed. Birth weight, height, head circumference, and tricep and subscapular skinfold thickness measurements were recorded. Secondly, a home visit was conducted after the infant had reached four weeks of age - during the fifth postnatal week. Premature infants ( $\leq 36$ weeks' gestation) were seen at 40 weeks postconceptional age. The home visit was deferred if the infant had not been discharged from hospital at least two weeks previously. A comprehensive questionnaire was administered. Data on postnatal smoking included active maternal smoking (cigarettes/day), whether the mother smoked in the same room as the infant or while feeding or holding the infant, number of other adults in the household who smoked, amount of smoking by other adults (total number of cigarettes/day) and whether the partner or other adults smoked in the same room as the infant or while feeding or holding the infant. If the mother or other smoked in a room when the baby was also physically occupying the same room then they were said to have smoked in the same room as the infant. Data were also obtained on the usual sleep position and pattern, body movement during sleep, infant illness and health service attendance, pattern and type of infant feeding, infant overnight clothing and bedding, home heating, ventilation and housing, and other characteristics of the infant and parental care. Anthropometric and temperature measurements were made on the infant and a developmental assessment was conducted. The physical features of the house were noted. Humidity and the 24 hour maxmin infant bedroom temperature on the day of home visit were recorded. Thirdly, a phone interview was conducted when the infant was 10 weeks of age, to review infant progress, illness history, feeding, and immunisation status.

In May 1991, findings from the study that infants who slept prone were at greater risk of SIDS were published ${ }^{10}$ and study participants were informed of this finding on how to reduce the risk of SIDS. By July 1991, the National SIDS Council of Australia had launched a national campaign on how to reduce the risks of SIDS, which included the health education message that infants should not be exposed to tobacco smoke (A Cahill 1993, personal communication). To examine the features of infant exposure to tobacco smoke in relation to the above, the two years before intervention (which began approximately on 1 May, 1991) ${ }^{11}$ were compared with the three subsequent years.

From 1 May 1988 until 30 April 1993, 6885 live born infants were eligible for inclusion in the survey. Of these eligible infants 6607 (96\%) and their mothers participated in the hospital interview and $6109(89 \%)$ participated in both the hospital and home interviews. The characteristics of the sample were as follows:

- $22 \%$ of infants were low birth weight $(<2500 \mathrm{~g})$

- $21 \%$ were born to teenage mothers;

- $69 \%$ of the infants were male;

- $12 \%$ were from multiple births;

- $33 \%$ of study births were in March-April;

- $26 \%$ were in May-July;

- $41 \%$ of study births were from AugustFebruary.

For the five year period beginning 1 May, 1988 the study recruitment system had a sensitivity of $47 \%$ and specificity of $79 \%$ with regard to SIDS cases under 12 months of age.

INFANT URINARY COTININE ANALYSIS

Infant urine samples were collected at home visit. The samples were collected during July and August 1991 by interviewers in the southern region by placing a urine specimen collection bag on the infant during the home interview. Thirty one infant urine samples were analysed for cotinine (a principal urinary metabolite of nicotine) by the Endocrinology Department, General Clinical Laboratories, North Sydney, using a double antibody ${ }^{125} \mathrm{I}$ radio immunoassay (DPC). The detected urinary cotinine concentrations ranged from $37.8 \mathrm{ng} / \mathrm{ml}$ to $221 \mathrm{ng} / \mathrm{ml}$. Urinary cotinine results were compared with questionnaire responses. Thirteen samples were collected from infants of mothers who smoked postnatally. All mothers who smoked stated that their children had been exposed to tobacco smoke in the preceding 48 hours. Of these samples, four were positive for cotinine and nine were not. In all positive samples, the mother sometimes or usually smoked in the same room as the infant. For the nine negative samples, only two of the smoking mothers stated that they never smoked in the same room as the infant. Eighteen samples were collected from mothers who did not smoke. One sample was positive. In this case the mother was a non-smoker but lived with a smoker who had smoked 50 cigarettes inside the house in the preceding 48 hours.

\section{STATISTICAL METHODS}

Smoking patterns in the cohort over time were examined using frequency tables and a one- 
Table 1 The relationship between parental, infant, or home factors and maternal smoking hygiene for mothers who smoke postnatally (outcome is mother does not smoke in same room where infant is)

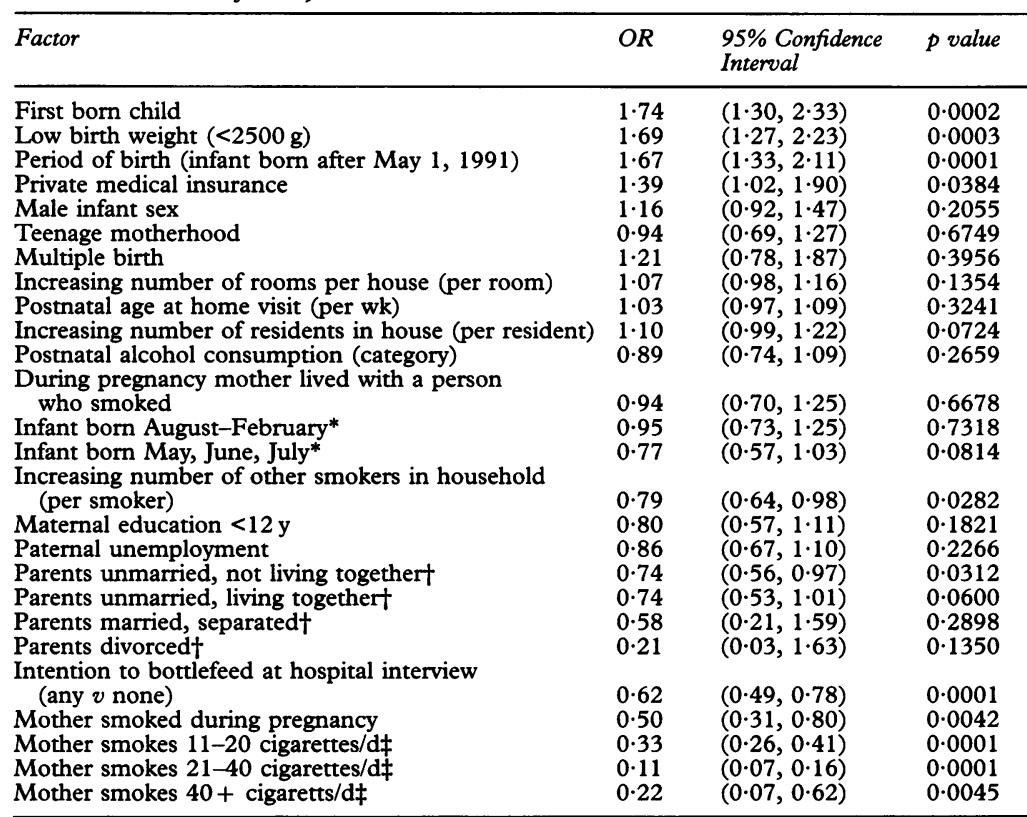

* Reference category = infants born March to April; $\nmid$ reference category = parents of infants are married and living together; $¥$ reference category $=$ mother smokes $1-10$ cigarettes smoked a day at home interview

Model also included terms for hospital of birth, duration of second stage of labour, and interviewer

sided $\chi^{2}$ test for trend. ${ }^{12}$ Logistic regression models ${ }^{13}$ were built to identify the factors independently associated with good smoking hygiene. Change in estimate methods were used to examine the effect of a potential confounder on an exposure disease association. In a statistical sense, a factor was said to confound an exposure-disease association if the risk ratio was altered by more than $10 \%$ after controlling for that individual factor. ${ }^{14}$ For mothers, the analysis was restricted to mothers who smoked

Table 2 The relationship between parental, infant, or home factors and the smoking hygiene of others in households where other residents smoke (outcome is others do not smoke in the same room as infant)

\begin{tabular}{|c|c|c|c|}
\hline Factor & $O R$ & $\begin{array}{l}95 \% \text { Confidence } \\
\text { Interval }\end{array}$ & $p$ value \\
\hline Low birth weight $(<2500 \mathrm{~g})$ & 1.79 & $(1 \cdot 38,2 \cdot 32)$ & 0.0001 \\
\hline Period of birth (infant born after May 1, 1991) & 1.65 & $(1.34,2.02)$ & 0.0001 \\
\hline First born child & $1 \cdot 30$ & $(1 \cdot 00,1 \cdot 70)$ & 0.0500 \\
\hline Private medical insurance & $1 \cdot 29$ & $(0.97,1.70)$ & 0.0759 \\
\hline Male infant sex & 1.02 & $(0 \cdot 83,1 \cdot 25)$ & 0.8566 \\
\hline Teenage motherhood & 0.86 & $(0.65,1.15)$ & 0.3102 \\
\hline Multiple birth & 0.98 & $(0.68,1.41)$ & 0.9147 \\
\hline Increasing number of rooms per house (per room) & 1.04 & $(0.97,1.13)$ & $0 \cdot 2812$ \\
\hline Increasing number of residents in house (per resident) & 1.06 & $(0.96,1 \cdot 16)$ & 0.2553 \\
\hline Postnatal age (per wk) & 1.02 & $(0.97,1.07)$ & 0.5447 \\
\hline $\begin{array}{l}\text { Increasing number of smokers in household } \\
\text { (per smoker) }\end{array}$ & 0.86 & $(0 \cdot 67,1 \cdot 10)$ & $0 \cdot 2273$ \\
\hline Intention of mother to bottlefeed at hospital & & & \\
\hline Infant born August to February* & 0.93 & $\begin{array}{l}(0.53,0.80) \\
(0.73,1.18)\end{array}$ & 0.5532 \\
\hline Infant born May, June, July* & 0.79 & $(0.61,1.02)$ & 0.0712 \\
\hline Parents unmarried, not living togethert & 0.80 & $(0.63,1.02)$ & 0.0702 \\
\hline Parents unmarried, living togethert & 0.89 & $(0.63,1 \cdot 26)$ & 0.5241 \\
\hline Divorced, separated, or other parental relationship $\dagger$ & 0.73 & $(0 \cdot 24,2 \cdot 19)$ & 0.5755 \\
\hline Maternal education $<12 \mathrm{y}$ & 0.72 & $(0.55,0.95)$ & $0 \cdot 0197$ \\
\hline Father unemployed at time of birth & $0 \cdot 83$ & $(0.66,1.05)$ & $0 \cdot 1136$ \\
\hline $\begin{array}{l}\text { Maternal cigarette smoking } \\
\text { (any } v \text { none at home interview) }\end{array}$ & 0.63 & $(0.52,0.76)$ & 0.0001 \\
\hline $\begin{array}{l}\text { Total amount smoked per day by other } \\
\text { adult residents }=11-20 \text { cigarettes } \ddagger\end{array}$ & 0.57 & $(0.45,0.71)$ & 0.0001 \\
\hline Total amount smoked per day by other adult & 0.31 & $(0.24,0.41)$ & 0.0001 \\
\hline $\begin{array}{l}\text { Total amount smoked per day by other } \\
\text { adult residents }=41+\text { cigarettes } \ddagger\end{array}$ & $0 \cdot 34$ & $(0.21,0.57)$ & 0.0001 \\
\hline
\end{tabular}

* Reference category = infants born March to April; † reference category=parents of infants are married and living together; $\neq$ reference category $=1-10$ cigarettes.

Model also included terms for hospital of birth, duration of second stage of labour, and interviewer. and the outcome indicator used to represent good smoking hygiene was a dichotomous variable which took the value ' 1 ' if the mother sometimes or usually smoked in the same room as the infant and ' 0 ' if the mother never smoked in the same room as the infant. The model included the terms used as components of the recruitment scoring system (table 1). Effect modification by period of birth was examined by building three term models with a term for period of birth, an individual factor, and a product term for period of birth and the factor being studied. ${ }^{13}$ To examine the relationship of smoking patterns during pregnancy and good postnatal smoking hygiene the term "mother lived with a smoker prenatally" was removed from the model and replaced with a separate term for smoking in each trimester ("any" vs "none") or a term for the average daily duration of maternal exposure to tobacco smoke during pregnancy. Contingency tables and the MantelHaenzel $\chi^{2}$ test were used to examine the relationship between maternal smoking hygiene and home ventilation habits. A two tailed Fisher exact test ${ }^{15}$ was used if a cell contained five or fewer observations. A multivariate logistic regression model was also built for other residents who smoked. The outcome factor was a binary term which took the value " 1 " if others sometimes or usually smoked in the same room as the infant and " 0 " if others never smoked in the same room as the infant. This is shown in table 2 .

\section{Results}

The overall proportion of cohort mothers who smoked during pregnancy or postnatally did not decline during the study period (fig 1 ). The proportion of mothers who smoked during the second and third trimesters of pregnancy decreased over time (fig 1 ). In addition, the level of smoking hygiene around the infant improved significantly over time (fig 1 ). That is, a positive trend was observed for each of the three parameters used to examine maternal and also other smoking hygiene. Although this is encouraging, it should be noted that for mothers who smoke, the proportion of mothers never smoking in the same room as the infant in the most recent year was still only $29 \%$. The amount smoked by mothers postnatally also varied by year $(p=$ 0.003 ). For both mothers and others, the proportion with good smoking hygiene increased with increasing intimacy with the infant. For example, in each year, the proportion of mothers who smoked in the same room as the baby was higher than the proportion of mothers who smoked while holding the baby which, in turn, was higher than the proportion of mothers who smoked while feeding the baby. Maternal smoking hygiene was examined for different levels of maternal smoking. The level of maternal smoking hygiene was inversely related to the amount smoked (fig 2). The proportion of mothers who did not smoke in the same room as the baby was significantly higher in the period from May 1, 1991 compared with the two earlier years $(\mathrm{OR}=1.56(1.31,1.87))$. After adjustment for paternal unemployment, 


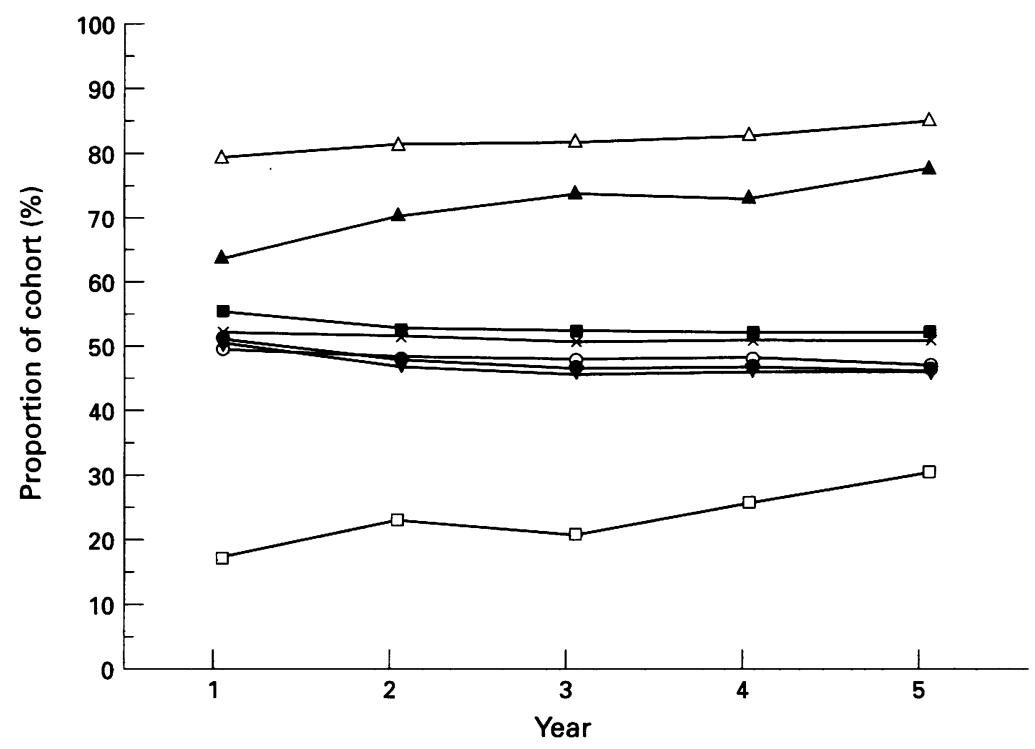

Figure 1 Smoking patterns of mother in relation to year of birth. ( $p$ value for test of trend over the five year period.)

- Maternal smoking during pregnancy $p=0.087$

$-\times-$ Maternal smoking - trimester $1 p=0 \cdot 2381$

- Maternal smoking - trimester $2 p=0.008$

- $\triangle$ - Maternal smoking - trimester $3 p=0.007$

- - Maternal smoking postnatally $p=0.093$

$-\square-$ Mother not smoking while in same room (smoking mothers only) $p=<0.0001$

- Mother not smoking while holding infant (smoking mothers only) $p=<0.0001$

$-\triangle-$ Mother not smoking while feeding infant (smoking mothers only) $p=0.046$

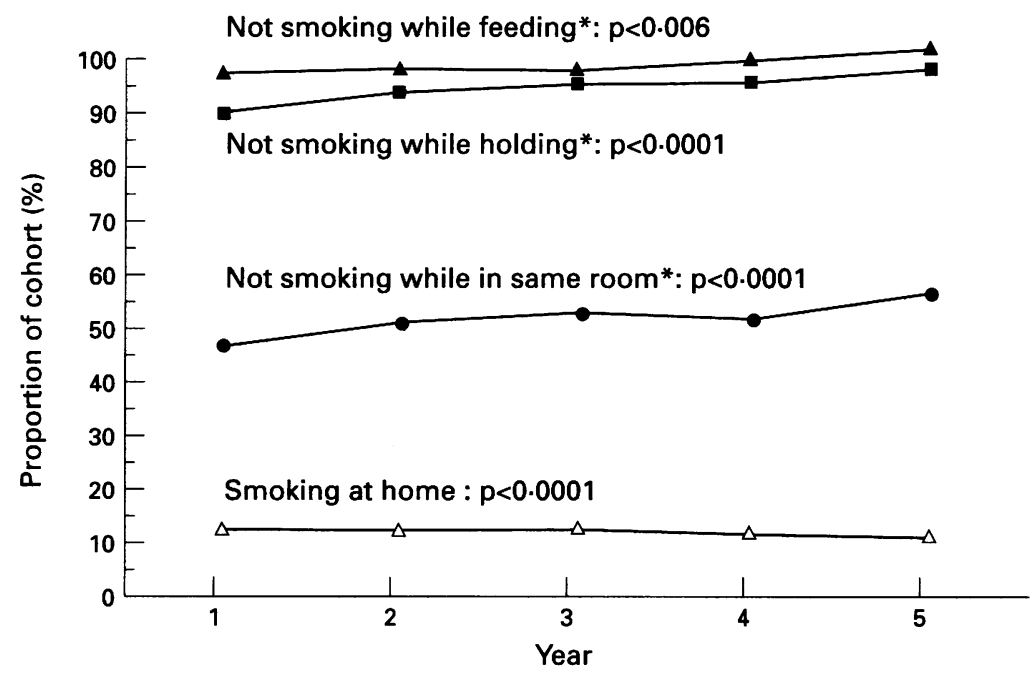

Figure 2 The infant cohort: smoking patterns of others by year of birth. * Whole cohort. $p$ value for test of trend over five years.

which became more common over time and was inversely associated with good maternal smoking hygiene (unadjusted $O R=0.59(0.48$, $0 \cdot 72)$ ), the beneficial effect of being born after May 1, 1991 was increased (adjusted $\mathrm{OR}=$ $1 \cdot 75(1 \cdot 46,2 \cdot 11))$.

None of the other variables listed in table 1 altered the univariate risk estimate for period of birth by more than $10 \%$. Multiple logistic regression analysis was conducted to identify factors independently associated with good maternal smoking hygiene, after adjustment for confounding factors (table 1). Factors positively associated with good smoking hygiene were being born after May 1, 1991, being a firstborn child, low birth weight $(<2500 \mathrm{~g})$ and having private health insurance. Factors in- versely associated with good smoking hygiene were higher levels of cigarettes smoked per day, maternal smoking during pregnancy, and intention to bottle feed at hospital (any $v$ none). In addition, the form of the relationship between the child's parents (married living together, unmarried living together, married but separated or divorced) was significantly related to maternal smoking hygiene. The association between the factors listed in table 1 and maternal smoking hygiene did not vary by period of birth. The following factors were not significantly associated with good smoking hygiene in the multivariate analyses summarised in table 1: infant sex, maternal age, multiple birth, postnatal age, season of birth, maternal education, paternal unemployment during pregnancy, mother living with a person who smoked, number of residents in house, and postnatal alcohol consumption.

Smoking during pregnancy was then examined by trimester. Maternal smoking during the first trimester of pregnancy was associated with poor smoking hygiene $(\mathrm{OR}=0.77(0.66$, $0.88)$ ). Smoking during the second $(O R=0.66$ $(0.58,0.78))$ or third $(\mathrm{OR}=0.71(0.61,0.82))$ was also associated with poor postnatal smoking hygiene in this multivariate analysis. To examine the role of passive smoking during pregnancy further, the term for "living with a smoker during pregnancy" was replaced by terms for the average number of minutes per day the mother was exposed to tobacco smoke during pregnancy inside the home and outside the home. Mothers who were exposed to smoke for longer periods during pregnancy were less likely to have good smoking hygiene around the infant postnatally $(\mathrm{OR}=0.87(0.83,0.91))$ for in home exposure and $(O R=0.92(0.87$, $0.97)$ ) for outside home exposure.

Maternal smoking hygiene was examined in relation to home ventilation habits. No association was found between good smoking hygiene and the opening of windows during different weather conditions $(p=0 \cdot 198)$, the opening of windows in the infant's room at night $(p=0.062)$ or the opening of the door of infant's room at night $(p=0.719)$. Good smoking hygiene was inversely related to air freshener use in the infant bedroom - only $12 \cdot 1 \%(25 / 206)$ of the smoking mothers who used air freshener in the baby's room smoked in the room where their baby was compared with $23 \cdot 1 \%(6034 / 2613)$ of the smoking mothers who did not use air freshener in this way $(\mathrm{p}=0.0007)$.

The smoking hygiene of others around the baby was examined by multivariate logistic regression for those other than the mother who smoked and lived with the baby (table 2). Controlling for other terms in the model, the effect of being born after May 1, 1991 was to increase the likelihood that others would not smoke in the same room as the infant. Infants whose mothers smoked were more likely to have others smoking near them (that is, in the same room). The amount smoked by others was inversely related to good smoking hygiene (table 2). Again, the association between the 
factors and smoking hygiene of others around the baby did not vary by period of birth.

\section{Discussion}

The proportion of mothers smoking during the first trimester or postnatally did not decrease significantly during the time period of the study. However the proportion of mothers smoking during the second or third trimester did decline over time, suggesting antenatal smoking cessation advice is having some impact, at least during pregnancy. The level of smoking for Australian women is of concern. A national report examining age standardised smoking trends in Australia showed that the prevalence of smoking had fallen, particularly among men, between 1976 and 1989, with the prevalence of smoking among women not significantly different to that of men in the last of the six surveys. ${ }^{16}$ Our centre has recently reported that the incidence of lung cancer in young Tasmanian women (25-44 years) was higher than their male counterparts during the period 1983 to $1992 .{ }^{17}$ Thus, the level of smoking among women of child bearing age remains a large public health problem.

The data do, however, show an improvement over time in the smoking hygiene of mothers who smoke and of other smokers in relation to smoking near infants. Although these trends are encouraging, it should be noted that even for the most recent year of data, two thirds of mothers who smoked sometimes or usually smoked in the same room as their infant, suggesting a good deal of further improvemen is required before the goal of a tobacco free environment for children ${ }^{5}$ is achieved.

The cohort study has collected standard information over time and has a high response rate - minimising selection bias. Although the smoking data are based on self report, reporting bias is unlikely to be an explanation of smoking hygiene findings in this study for several reasons. Urinary cotinine has been found to be highly correlated $(p<0.0001)$ with the parental report of exposure to tobacco smoke. ${ }^{18}$ Salivary cotinine levels have previously been shown to relate well to self reported smoking in an adult Australian sample $(n=1177)$, with a sensitivity of $92.6 \%$ and specificity of $93.4 \% .^{19}$ In addition, in the small validation study conducted here, no detectable urinary cotinine was found in the urine of infants whose mothers reported that they had not been exposed to tobacco smoke in the preceding 48 hours. However, the cotinine assay used here was less sensitive than other reported assays. ${ }^{1820}$ Overall, the urinary cotinine assay had a sensitivity of $31 \%$ and specificity of $94 \%$ in relation to maternal smoking. We plan to repeat the smoking validation facet of this study using a larger sample and a more sensitive assay.

This cohort is not a representative sample of live births. However, in order for the results of an analytical cohort study to be generalisable to other populations it is not necessary for the cohort to be representative of the community from which it was selected. ${ }^{2122}$ Mietinenen states that the key concerns with regard to a study base are that the eligible participants are well defined, that there is a good distribution of determinants, confounders, and modifiers in the study and adequate sample size. ${ }^{22}$ This cohort fulfills these criteria. Therefore we do not consider that the lack of representativeness of the cohort, in itself, affects external validity. However, the generalisation of these results to other settings should include an assessment of how similar the target population is to this cohort with regard to maternal and other behaviours.

Mothers whose infants were born after May 1, 1991 had better smoking hygiene habits, even after adjustment for socioeconomic factors, the components of the scoring system used to define cohort eligibility, and other factors such as amount smoked postnatally. The improvement in smoking hygiene over time could not be explained by changes over time of any of the factors listed in table 1. The improvement of parental smoking hygiene over time can be attributed partly to health education activities with regard to smoking hygiene, such as the national campaign to "Reduce the risk of SIDS". The finding that the amount smoked is inversely related to good smoking hygiene for mothers and others is of concern, because the more a person smokes, the more important it is that she or he should have good smoking hygiene near infants. Low birth weight of the infant was associated with better smoking hygiene in the mother. This may be because the mothers of low birth weight infants considered them to be more vulnerable. Mothers who smoked postnatally were more likely to have good smoking hygiene if the infant was their first born. However, we did not find that mothers of first born infants had a greater improvement in smoking hygiene over time than the mothers of children of higher birth order. Mothers and others in the household of infants who were born during the cooler months of May, June, and July tended to be less likely to have good smoking hygiene presumably because the cold weather discouraged smoking outside or in another part of the house to where the infant was located.

Maternal smoking during pregnancy was associated with poorer smoking hygiene, even after adjustment for confounders such as amount smoked (table 2). This finding may partially reflect reporting bias - with honest smoking mothers more likely to admit to smoking during pregnancy and also to smoking in the same room as their baby. We explored the effect of such differential misclassification on the association between smoking during pregnancy and good smoking hygiene (unadjusted $\mathrm{OR}=0.30 \quad(0.20,0.46))$ using four assumptions. Firstly, that the misclassification of smoking during pregnancy and smoking hygiene was as extreme as $15 \%$; that mothers who did not report smoking during pregnancy were twice as likely to falsely report good smoking hygiene; that mothers who reported good smoking hygiene were twice as likely to falsely report that they didn't smoke during pregnancy, and that falsely reporting on both smoking during pregnancy and smoking hygiene was 
twice as common as a single false report. Even with this extent of misclassification, smoking during pregnancy remained inversely associated with good smoking hygiene $(\mathrm{OR}=$ $0.50(0.30,0.82))$

Interestingly, mothers who smoked postnatally and were also passively exposed to more tobacco smoke antenatally were also likely to have poor smoking hygiene. This could suggest a subculture of high environmental tobacco smoke exposure or that these mothers were tolerant of a smoking atmosphere and did not perceive it as an important health risk for their child in utero or postnatally. The cohabiting arrangement of the parents was related to maternal smoking hygiene. Smoking mothers with good smoking hygiene were more likely to use airfreshener in the baby's bedroom than those with poor smoking hygiene. It is likely that these mothers were well intentioned but not aware that a more appropriate action would be to ventilate the baby's room well.

The smoking hygiene of "others" was requested rather than "father" because the cohort sample has many young mothers and only $57 \%$ of the cohort mothers were married. It was our experience that the maternal grandmother is often a significant carer and visitor to young, single mothers and the question was broadened to include people such as this. The smoking hygiene of others around the infant was also found to improve over time. Again, an inverse relationship was found between amount smoked and good smoking hygiene. Not surprisingly, the smoking hygiene of others around the baby was better if the baby's mother did not smoke. Non-smoking mothers would be more likely to perceive environmental tobacco smoke as hazardous and hence instruct others not to smoke around baby.

This report centres on the smoking hygiene of mothers and others. Knowledge of factors associated with good smoking hygiene and trends over time should lead to health benefits for the infant. The United States Environmental Protection Agency has concluded, after an extensive review of scientifc evidence, that postnatal exposure to environmental tobacco smoke as well as maternal smoking during pregnancy can predispose a child to a variety of respiratory effects that can, themselves, have long term consequences. ${ }^{23}$ In a recent casecontrol study in southern California, the adjusted odds ratio for SIDS associated with passive smoking from the mother, father, and other sources was $3.50(1 \cdot 81,6 \cdot 75)$ after adjustment for maternal smoking during pregnancy, birth weight, sleep position, prenatal care, medical conditions at birth, and breast feeding. ${ }^{24}$ In a recent, large cross sectional study, Cook et al investigated the relationship of individual, family and community factors to passive exposure to tobacco smoke in children aged 5-7 years, using salivary cotinine concentration as a biological marker of exposure. The geometric mean for salivary cotinine rose from $0.29(0.28,0.31) \mathrm{ng} / \mathrm{ml}$ in children with no identified exposure to cigarette smoke to $4.05(3.71,4.42) \mathrm{ng} / \mathrm{ml}$ if both parents smoked. The cotinine concentration was strongly related to the number of smokers to whom the child was usually exposed. ${ }^{20}$ They confirmed that parental smoking was the most important determinant of tobacco exposure in this group, with maternal smoking of greater importance than paternal smoking. ${ }^{20}$ Children not exposed to tobacco smoke at home had low cotinine concentrations, the level depending on the prevalence of smoking in the community. ${ }^{20}$

Infant cotinine levels have also been studied in relation to respiratory disease. Reese et al found significantly higher levels of urinary cotinine in children hospitalised for broncholitis than controls. ${ }^{25}$ Chilmonczyk et al found that among a group of 199 asthmatic children, acute exacerbations of asthma during the preceding year were significantly more common among the children with high urinary cotinine levels (>39 $\mathrm{ng} / \mathrm{ml}$ ) compared with those with low cotinine levels $(<10 \mathrm{ng} / \mathrm{ml})$, with an odds ratio of $1 \cdot 7(1 \cdot 4,2 \cdot 1)$ after adjustment for maternal age and other confounders. ${ }^{2}$

The Substance Abuse Committee of the American Academy of Pediatrics recommends that inquiry into tobacco use and smoke exposure should be a routine part of both the prenatal visit with parents and every appropriate pediatric health visit. ${ }^{5}$ Furthermore, smoking parents should be informed of the dangers of environmental tobacco smoke exposure and the implications and complications of exposing their children to tobacco smoke. ${ }^{5}$ Information about available smoking cessation assistance should be offered. ${ }^{5}$ In conclusion, the findings from this cohort study are that maternal smoking rates have not decreased substantially and a large proportion of parents who smoke still smoke in the same room as their infant. However, parental smoking hygiene around the infant has significantly improved over time. This study's identification of parental and infant factors associated with good smoking hygiene should assist health education planning.

Supported by the National Institutes of Health, USA (grant no = HP 28979-02), COSP, Department of Human Services and Health, National Sudden Infant Death Syndrome Council of Australia and constituent organisations, Tasmanian State Government, Australian Rotary Health Research Fund, National Health and Medical Council of Australia, Zonta International, Wyeth Pharmaceuticals, and Tasmanian Sanatoria After-Care Association

We are indebted to the parents and infants who participated in the cohort study; $K$ Evans, $H$ Donaldson, $M$ Smiley and other interviewers for data collection; J Cochrane for data management; to all the oranisations and practitioners involved in SIDS risk for the in SIDS risk factor health education activities; to the Departments of Hospital Pathology, Police, and Justice for their ctudy. We than F Bo hort assays.

1 Environmental Protection Agency (EPA). Respiratory health Washington, DC: Office of Research and Der disorders. Office of Air and Rafice of Research and Development

2 Chilmon of sociation between expo Lure to environ KN, et al. Association Med 1993;328:1665-69.

Mitchell EA, Ford RPK, Stewart AW, et al. Smoking and the sudden infant death syndrome. Pediatrics 1993;91: the sudde.

4 Schoendorf KC, Kiely JL. Relationship of sudden infant death syndrome to maternal smoking during and after pregnancy. Pediatrics 1992;90:905-8.

5 Committee on Substance Abuse, American Academy of Pediatrics. Tobacco-free environment: an imperative for the health of children and adolescents. American Academy of Pediatrics Committee on Substance Abuse. Pediatrics 1994;93:866-8. 
6 Charlton A. Children and passive smoking: a review. The fournal of Family Practice 1994;38:267-77.

7 Matsuki H, Kasuga H, Osaka F, Yanagisawa Y, Nishimura H. A comparative study on the health effects of smoking H. A comparative study on the health effects of smoking
and indoor air pollution in summer and winter. Tokai $\mathscr{f}$ Exp Clin Med 1985;10:427-37.

8 Greenberg RA, Haley NJ, Etzel RA, Loda FA. Measuring the exposure of infants to tobacco smoke. Nicotine and cotinine in urine and saliva. $N$ Engl f Med 1984;310: 1075-8.

9 D'Espaignet ET, Dwyer T, Newman NM, Ponsonby AL, Candy S. The development of a model for predicting infants at high risk of sudden death syndrome in Tasmania. Paediatric and Perinatal Epidemiology 1990;4:422-35.

10 Dwyer T, Ponsonby AL, Newman NM, Gibbons LE. Prospective cohort study of prone sleeping position and sudden infant death syndrome. Lancet 1991;337:1244-7.

11 Ponsonby AL, Dwyer T, Kasl SV, Cochrane JA, Newman NM. An assessment of the impact of public health activities to reduce the prevalence of the prone sleeping position during infancy: The Tasmanian cohort study. Prev Med 1994;23:402-8.

12 Fisher LD, van Belle G. Biostatistics. A methodology for the health sciences. New York: John Wiley, 1993:253-255.

13 Armitage P, Berry G. Statistical methods in medical research. 3rd Ed. Oxford: Blackwell Scientific Publications $1994 ; 422-36$

14 Greenland S. Modeling and variable selection in epidemiologic analysis. Am F Public Health 1989;79:340-9.

15 Randles RH, Wolfe DA. Introduction to the theory of nonparametric statistics. New York: John Wiley 1979;349-50.
16 Hill DJ, White VM, Gray NJ. Australian patterns of tobacco smoking in 1989. Med $\mathcal{f}$ Austr 1991;154:797-801.

17 Dwyer T, Blizzard L, Shugg D, Hill D, Ansari MZ. Highe lung cancer rates in young women than young men lung cancer rates in young women than young men: 5:351-8.

18 Schneider JM, Capolaghi B, Briancon S, Covi G, Merlin JP, Leveau PH. Passive smoking in children. Its detection by the assay of urinary cotinine. Arch Fr Pediatr 1993 50(7):567-571.

19 Pierce JP, Dwyer T, DiGiusto E, et al. Cotinine validation of self-reported smoking in commercially run community surveys. F Chron Dis 1987;40:689-95.

20 Cook DG, Whincup PH, Jarvis MJ, Strachan DP, Papacost $\mathrm{O}$, Bryant A. Passive exposure to tobacco smoke in children aged 5-7 years: individual, family, and community factors. BMf 1994;308:384-9.

21 Rothman KJ. Modern epidemiology. Boston: Little, Brown \& Co, 1986:95-6.

22 Mietinenen OS. Theoretical epidemiology: principles of occurrence research in medicine. USA. New York: John Wiley, 1985

23 Jinot J, Byard S. Respiratory health effects of passive smoking: EPA's weight-of-evidence analysis. $\mathcal{F}$ Clin Epidemiol 1994;47:339-49.

24 Klonoff-Cohen HS, Edelstein SL, Lefkowitz ES, et al. The effect of passive smoking and tobacco exposure through breast milk on sudden infant death syndrome. $\mathfrak{F} A M A$ 1995;273:795-8.

25 Reese AC, James IR, Landau LI, Lesouef PN. Relationship between urinary cotinine level and diagnosis in childre admitted to hospital. Am Rev Respir Dis 1992;146:66-70. 